Chem. Phys. Lett.

\title{
Laser Synthesis and Spectroscopy of Acetonitrile/Silver Nanoparticles
}

\author{
S. T. Akin, X. Liu and M. A. Duncan* \\ Department of Chemistry, University of Georgia, Athens, Georgia 30602, U.S.A.
}

\begin{abstract}
Silver nanoparticles with acetonitrile ligands are produced in a laser ablation flow reactor. Excimer laser ablation produces gas phase metal clusters which are thermalized with helium or argon collisions in the flowtube, and reactions with acetonitrile vapor coordinate this ligand to the particle surface. The gaseous mixture is captured in a cryogenic trap; warming produces a solution of excess ligand and coated particles. TEM images reveal particle sizes of 10-30 nm diameter. UV-visible absorption and fluorescence spectra are compared to those of standard silver nanoparticles with surfactant coatings. Deep-UV ligand absorption is strongly enhanced by nanoparticle adsorption.
\end{abstract}




\section{Introduction}

Small silver nanoclusters have fascinating optical properties, important in a variety of spectroscopy and imaging applications $[1,2]$. The absorption of small silver clusters and their ions has been studied in the gas phase or isolated in rare gas matrices [3-6]. Ultra-small silver clusters bind to DNA and serve as fluorescence markers [7-11]. When formed as larger nanoparticles, silver exhibits a strong plasmon resonance which is the basis for surface-enhanced Raman spectroscopy [12-14]. Plasmon resonances in silver nanoparticles have been employed to enhance light harvesting efficiencies in dye-sensitized solar cells $[15,16]$. The size dependence of the plasmon resonance as a function of particle size has been investigated with both experiments [3-6] and theory [17-19]. Most silver nanoparticles are produced by solution synthesis, in which metal reduction is accompanied by surfactant coating [1,2,20-22]. Other studies have produced nanoparticles by chemical or physical vapor deposition, by laser vaporization in solution, or by laser vaporization coupled to electrospray ionization [23-25]. In the present report, silver nanoparticles are produced in the gas phase by laser vaporization, followed by small-molecule (acetonitrile) ligand coating with reactions in a gas flow reactor. We investigate the size distribution produced and the effect of the small-molecule ligand coating on the spectroscopy of these particles.

Silver clusters in the size range of 10's of atoms begin to develop the plasmon electronic structure known for the bulk [3-6]. The size-dependence of the plasmon resonance has been investigated extensively using Mie theory or its variants and is well understood [3-6,17-19]. Ligand interactions are often critically important in determining optical spectra. For small and intermediate-sized clusters, certain ligands have allowed atom-specific cluster sizes to be isolated and their size-dependent optical properties to be measured [7-11,26-30]. In larger systems, the ligand interactions may appear as specific spectral effects, as charge transfer interactions, or as a 
dielectric influence on the plasmon in different systems [31-34]. However, synthetic schemes employed to date generally do not allow small-molecule ligands to be employed for these systems. The laser-based methods here allow a representative small-molecule, acetonitrile, to be employed for ligand coating. Here, we investigate its effect on cluster production and optical properties.

\section{Experimental}

Ligand-coated silver nanoparticles are produced in the gas phase by laser ablation/vaporization [35] in a flow reactor described previously [36,37]. A rotating silver rod is ablated with the output $(80-100 \mathrm{~mJ} /$ pulse $)$ of a high repetition rate $(400 \mathrm{~Hz})$ excimer laser (Lambda Physik LPX Pro 240) at the $\mathrm{KrF}$ wavelength (248 nm) in a continuous flow of helium or argon gas. The pressure in the flowtube is about 10 torr. Acetonitrile vapor above a liquid sample heated to $50^{\circ} \mathrm{C}$ is injected into the gas flow at a point $30 \mathrm{~cm}$ downstream from the ablation point. The flowing gas mixture is trapped at liquid nitrogen temperature in a roundbottomed flask equipped with glass beads for increased gas contact with cold surface area. The trapped material resulting from a two-hour run is collected and then allowed to warm gradually to room temperature, producing a clear, slightly yellow solution. The solution is investigated with electrospray ionization (ESI) time-of-flight mass spectrometry (PerSeptive Biosystems, Mariner), UV-visible absorption spectroscopy (Shimadzu UV-2600), fluorescence spectroscopy (Shimadzu RF-5301C), and transmission electron microscopy (FEI Technai 20). Particle size distributions from five selected TEM images were measured with ImageJ software [38]. 


\section{Results and discussion}

A two-hour run using the laser ablation flow reactor and the conditions indicated above produces a light yellow solution in which acetonitrile acts as both the ligand and the solvent. The synthesis is reproducible on different days and is not particularly sensitive to the vaporization laser power employed. The solution produced is stable for long periods (months) with or without refrigeration. It can be dried out to a powder and then re-dissolved in acetonitrile, but is not soluble in water (particles immediately aggregate). The concentration of the solution can be estimated from the plasmon intensity in the UV-visible spectrum (see below) and the estimated particle size (taken from the plasmon position while assuming bulk silver density) of $4 \times 10^{8} \mathrm{amu}$. On the basis of this analysis, we produce approximately $0.8 \mathrm{mg}$ of sample in a two-hour synthesis run. According to previous work on this apparatus [36,37], cluster growth is believed to occur in the gas phase in the flow tube. Consistent with this, variation of buffer gas flow, or ligand partial pressure affect the sample production, but there is no further aggregation or growth of particles in solution when samples sit for extended periods of time (weeks).

Figure 1 shows the ESI mass spectra measured for the acetonitrile-coated nanoparticles (Ag-MeCN NP) produced here compared to that for a "standard" silver nanoparticle (Ag NP) sample of $50 \mathrm{~nm}$ silver nanoparticles coated with citrate [39]. In both cases, the ESI process produces small gaseous silver masses, but there are no masses produced corresponding to nanosized clusters. Assuming bulk silver density, the $50 \mathrm{~nm}$ particles should have a mass of roughly $4 \times 10^{8} \mathrm{amu}$, and therefore it is understandable that the singly charged parent ions of these particles are not detected. The small molecular weight ions produced then presumably arise either from the presence of small silver clusters in solution, or from sputtering/ablation of larger particles in the ESI discharge. The new sample produced here exhibits more small silver-atom 
cluster masses with attached acetonitrile, whereas the citrate-capped Ag NP sample produces mostly atomic silver with attached acetonitrile. The acetonitrile sample may contain more smaller clusters from the laser vaporization process. However, it is also possible that the new sample ablates metal more easily than the citrate-capped Ag NP sample because of its different ligand coating and the likely weaker coordination of acetonitrile compared to citrate. Metal-CN masses are present in these spectra, but we believe these come from fragmentation in the electrospray ionization process. It is difficult to rule out $\mathrm{CN}$ as a ligand here, but other studies of acetonitrile on more reactive metals and metal oxides under more gentle laser desorption mass spectrometry conditions do not find CN adducts [37].

Figure 2 shows a TEM microscopy image of the Ag-MeCN NP sample. The left frame shows an island of particles produced after solvent evaporation on the probe, whereas the right frame shows an expanded view of a single particle. These images are typical for different samples from a single batch of synthesized material, or for different batches of material produced in different runs on different days. Figure 3 shows a histogram of particle sizes determined from five selected TEM images using the ImageJ software [38]. Although the particles are clearly not monodisperse, most have sizes of 10-30 nm diameter. At the resolution of this TEM, the particles appear to be roughly spherical with no evidence for faceting.

Figure 3 shows the UV-visible absorption spectroscopy of the Ag-MeCN NP sample compared to that of the citrate-capped Ag NP sample. Both samples are studied in acetonitrile solutions; a spectrum of the acetonitrile solvent is also included for comparison. As shown in the right side of the figure in red, the citrate-capped particles have a plasmon resonance at $421 \mathrm{~nm}$, at the position expected for this size particle. Compared to this, the Ag-MeCN NP sample produced in the LVFR (blue) exhibits a resonance at $403 \mathrm{~nm}$, typical for silver nanoparticles in the range of slightly smaller particles. Mie theory calculations for a $25 \mathrm{~nm}$ spherical silver 
nanoparticle in acetonitrile predict a plasmon at $402 \mathrm{~nm}$ [40]. Mie theory calculations predict no shift in the plasmon resonance when a layer of acetonitrile ligand is added to the $25 \mathrm{~nm}$ silver sphere [40]. The observed plasmon resonance for our Ag-MeCN NPs is relatively broad compared to that of the $50 \mathrm{~nm}$ citrate-capped Ag NPs, which is understandable for a sample with a wider particle size distribution.

The left side of Figure 3 shows the shorter wavelength region of the spectrum, which is rarely reported in the literature for silver nanoparticles. Here, the citrate-capped Ag NP sample has a peak at $192 \mathrm{~nm}$. Sodium citrate in water absorbs at this wavelength, and so this peak is attributed to the citrate on the surface of the silver nanoparticles. The spectrum of the Ag-MeCN particles exhibit a strong absorption around $197 \mathrm{~nm}$, that falls off gradually to longer wavelengths. Also shown in black is the spectrum in this region for the pure acetonitrile solvent, which has a strong resonance at about $188 \mathrm{~nm}$. This solvent absorption was background subtracted from that of the Ag-MeCN NPs. The remaining absorption at $197 \mathrm{~nm}$ can then be assigned to the acetonitrile ligands attached to the silver particles. The interaction with the silver particle surface shifts this resonance significantly to the red from that of the pure acetonitrile. Silver nanoparticles have been produced previously by laser ablation of a sample submerged in liquid acetonitrile [41]. However, the particles produced in this way were assumed to be ligandfree, consistent with the plasmon resonance occurring at its predicted position for the naked metal system. The deep-UV spectra presented here show clearly that acetonitrile is acting as a ligand having a strong interaction with the silver surface. This is consistent with the solubility of these nanoparticles. However, this metal-ligand interaction apparently does not shift the plasmon appreciably, consistent with the earlier work [41].

The deep-UV absorption from the acetonitrile ligand is extremely intense, roughly approximating that of the pure acetonitrile solvent, which of course has a much higher 
concentration. It is therefore interesting to investigate this intensity further. Paramelle and coworkers have described a method employing the plasmon resonance intensity to determine the concentration of silver nanoparticles in solution [42]. Using the extinction coefficient for the silver particles at the plasmon resonance, we derive a concentration of $1.02 \times 10^{-11} \mathrm{M}$ for the silver particles in our sample. Using the representative size of $25 \mathrm{~nm}$ for the particle size, an area of $0.4 \mathrm{~nm}^{2}$ for the surface area of an acetonitrile ligand, and assuming full surface coverage (because the particles are in acetonitrile solvent) we calculate that there are about 5,300 ligands per particle, for an acetonitrile-ligand concentration of $5.4 \times 10^{-8} \mathrm{M}$. Using this concentration and the acetonitrile absorbance at $197 \mathrm{~nm}$, we determine an extinction coefficient of $2.1 \times 10^{7}$ $\mathrm{cm}^{-1} \mathrm{M}^{-1}$. This compares to the value of $5.7 \times 10^{-2} \mathrm{~cm}^{-1} \mathrm{M}^{-1}$ for pure acetonitrile, and represents a remarkable surface enhancement for the ligand absorption. If the surface coverage is incomplete, this value would be higher. Enhancement of the absorption strength of dye molecules adsorbed on silver nanoparticles has been reported previously $[15,16]$, but the enhancement wavelengths were those in resonance with the silver surface plasmon. Such enhancements of deep-UV absorption has not been reported previously to our knowledge. This spectral enhancement is also consistent with a significant silver-acetonitrile metal-ligand interaction.

Figure 4 shows the emission spectra of our Ag-MeCN NPs compared to that of the citrate-coated particles. Intensities are normalized to concentrations in these spectra [42]. The right frame in the figure shows the visible emission from excitation at $400 \mathrm{~nm}$. In this region, the expected plasmon emission occurs, which is quite similar in position for both the citrate-capped nanoparticles and our acetonitrile species. The citrate-coated particles have slightly more intense emission. The left frame of the figure shows the ultraviolet region of the spectrum, with 
excitation at $220 \mathrm{~nm}$. Here the emission for both kinds of particles are more intense than they are in the longer wavelength region (see scale change). Both the citrate-coated and acetonitrilecoated NPs exhibit an emission feature at $370 \mathrm{~nm}$, with that of the latter more intense. The acetonitrile-coated particles have an additional feature at $305 \mathrm{~nm}$, which is not found for the citrate-coated particles. The assignment of these UV emission is not straightforward, as this region of the spectrum is frequently not reported in nanoparticle studies. Because the citratecapped $\mathrm{Ag} \mathrm{NP}$ emission is relatively weak at $370 \mathrm{~nm}$, we initially thought that this might be caused by the more limited surface contact of the citrate-coated particles with the acetonitrile solvent. However, this emission at $370 \mathrm{~nm}$ is observed in both acetonitrile and water solvents, and therefore must be associated with the silver itself, rather than an acetonitrile-surface effect, in these systems. This emission is three to five times more intense for Ag-MeCN NPs than it is for the citrate-capped Ag NPs. The $305 \mathrm{~nm}$ emission is seen only for the acetonitrile-capped particles. Neither acetonitrile nor citrate are responsible for this peak, as acetonitrile shows only a small emission around $240 \mathrm{~nm}$, while citrate exhibits no emission in this region. It is conceivable that this emission may be due to metal-ligand charge transfer, as this has been observed for other noble metal nanoparticles in solution [43]. However, another strong possibility is that both the 370 and $305 \mathrm{~nm}$ emission features are from small ligand-coated silver clusters. Naked silver clusters in the 10-15 atom size range, studied in either the gas phase or in rare gas matrices, exhibit strong absorption resonances at about 3.3 and $4.0 \mathrm{eV}$, which correspond closely to the 370 and $305 \mathrm{~nm}$ emission features detected here [3-6]. These features are associated with the strongly blue-shifted plasmon resonances for these small clusters. It makes sense that either of the samples containing the citrate-capped or acetonitrile-capped particles might have such small clusters that would not be detected with low-resolution imaging methods. It also makes sense that these smaller clusters would be more abundant in the sample 
produced by laser vaporization. The presence of smaller clusters in our experiment would then also help to explain the mass spectra discussed earlier and shown in Figure 1. However, it is noteworthy that thiol-ligated clusters in this size range apparently do not produce such strong UV emission [30]. Apparently the acetonitrile ligand employed here perturbs the intrinsic plasmon emission in the smaller clusters to a lesser degree than other ligands, consistent with its negligible shift on the plasmon seen for the larger clusters. Future experiments will explore different laser ablation conditions and other similar ligands to attempt to make different sized silver particles and to further explore the strong UV emission in these samples.

\section{Conclusions}

Silver nanoparticles in the size range of $10-50 \mathrm{~nm}$ are produced with high repetition rate excimer laser vaporization and coated with acetonitrile ligands in the gas phase before their capture in solution. ESI mass spectrometry produces no large nanoparticle masses, but detects more small silver cluster cations for the acetonitrile-capped sample compared to that of the citrate capped Ag NPs. TEM reveals $10-50 \mathrm{~nm}$ particles, with an average particle size of about 10-20 nm. UV-Vis spectroscopy reveals "typical" plasmon features in both absorption and emission. However, a strong deep-UV absorption at $197 \mathrm{~nm}$ indicates a strong surfaceenhancement for acetonitrile ligands directly coordinated to the silver nanoparticles. Acetonitrile therefore binds strongly to the surface of these nanoparticles, providing their solubility. Its electronic spectrum is shifted, but it does not shift the particle plasmon resonances significantly. Additional strong emission features are seen for the acetonitrile-capped nanoparticles in the ultraviolet region at 370 and $305 \mathrm{~nm}$. This suggests the possible presence of small silver clusters not detected by microscopy. 


\section{Acknowledgments}

We gratefully acknowledge the generous support for this work from the Air Force Office of Scientific Research through grant no. FA9550-15-1-0088.

\section{References}

[1] U. Kreibig, M. Vollmer, Optical Properties of Metal Clusters, Springer-Verlag, Berlin, 1995.

[2] G. Schmid, ed., Nanoparticles, Wiley-VCH, Weinheim, Germany, 2004.

[3] J. Tiggesbäumker, L. Köller, H. O. Lutz, K. H. Meiwes-Broer, "Giant resonances in silver-cluster photofragmentation," Chem. Phys. Lett. 190 (1992) 42.

[4] J. Tiggesbäumker, L. Köller, K. H. Meiwes-Broer, A. Liebsch, "Blue shift of the Mie plasma frequency in Ag clusters and particles," Phys. Rev. A 48 (1993) R1749.

[5] M. Harb, F. Rabilloud, D. Simon, A. Rydio, S. Lecoultre, F. Conus, V. Rodrigues, C. Félix, "Optical absorption of small silver clusters: $\mathrm{Ag}_{\mathrm{n}},(\mathrm{n}=4-22), " \mathrm{~J}$. Chem. Phys. 129 (2008) 194108.

[6] S. Fedrigo, W. Harbich, J. Buttet, "Collective dipole oscillations in small silver clusters embedded in rare-gas matrices," Phys. Rev. B 47 (1993) 10706.

[7] C. I. Richards, S. Choi, J.-C. Hsiang, Y. Antoku, T. Vosch, A. Bongiorno, Y.-T. Tzeng, R. M. Dickson, "Oligonucleotide-stabilized Ag nanocluster fluorophores," J. Am. Chem. Soc. 130 (2008) 5038.

[8] B. Sengupta, C. M. Ritchie, J. G. Buckman, K. R. Johnsen, P. M. Goodwin, J. T. Petty, "Base-directed formation of fluorescent silver clusters," J. Phys. Chem. C 112 (2008) 18776. 
[9] S. Choi, R. M. Dickson, J. Yu, "Developing luminescent silver nanodots for biological applications," Chem. Soc. Rev. 41 (2012) 1867.

[10] J. T. Petty, S. P. Story, J. Hsiang, R. M. Dickson, "DNA-templated molecular silver fluorophores," J. Phys. Chem. Lett. 4 (2013) 1148.

[11] S. M. Copp, D. Schultz, S. Swasey, J. Pavlovich, M. Debord, A. Chiu, K. Olsson, E. Gwinn, "Magic numbers in DNA-stabilized fluorescent silver clusters lead to magic colors," J. Phys. Chem. Lett. 5 (2014) 959.

[12] S. J. Lee, Z. Guan, H. Xu, M. Moskovits, "Surface-enhanced Raman spectroscopy and nanogeometry: The plasmonic origin of SERS," J. Phys. Chem. C 111 (2007) 17985.

[13] B. Sharma, R. R. Frontiera, A.-I. Henry, E. Ringe, R. P. Van Duyne, "SERS: Materials, applications, and the future," Materials Today 15 (2012) 16.

[14] M. D. Sonntag, J. M. Klingsporn, A. B. Zrimsek, B. Sharma, L. K. Ruvuna, R. P. Van Duyne, "Molecular plasmonics for nanoscale spectroscopy," Chem. Soc. Rev. 43 (2013) 1230.

[15] N. C. Jeong, C. Prasittichai, J. T. Hupp, "Photocurrent enhancement by surface plasmon resonance of silver nanoparticles in highly porous dye-sensitized solar cells," Langmuir 27 (2011) 14609.

[16] J. Qi, X. Dang, P. T. Hammond, A. M. Belcher, "Highly efficient plasmon-enhanced dyesensitized solar cells through metal@oxide core-shell nanostructure," ACS Nano 5 (2011) 7108 .

[17] C. M. Aikens, S. Li, G. C. Schatz, "From discrete electronic states to plasmons: TDDFT optical absorption properties of $\operatorname{Ag}_{\mathrm{n}}(\mathrm{n}=10,20,35,56,84,120)$ tetrahedral clusters," J. Phys. Chem. C 112 (2008) 11272. 
[18] J. Zhao, A. O. Pinchuk, J. M. McMahon, S. Li, L. K. Ausman, A. L. Atkinson, G. C. Schatz, "Methods for describing the electromagnetic properties of silver and gold nanoparticles," Acc. Chem. Res. 41 (2008) 1710.

[19] L. Jensen, C. M. Aikens, G. C. Schatz, "Electronic structure methods for studying surface-enhanced Raman scattering," Chem. Soc. Rev. 37 (2008) 1061.

[20] M. Guzman, J. Dille, S. Godet, "Synthesis and antibacterial activity of silver nanoparticles against gram-positive and gram-negative bacteria," Nanomedicine: Nanotech. Bio. Med. 8 (2012) 37.

[21] A. Li, Q. Luo, S. Park, R. G. Cooks, "Synthesis and catalytic reactions of nanoparticles formed by electrospray ionization of coinage metals," Angew. Chem. Int. Ed. 53 (2014) 3147.

[22] S. Peglow, M.-M. Pohl, A. Kruth, V. Brüser, "Plasma based synthesis, electron microscopy, and optical characterization of Au-, Ag-, and Ag/Au-core-shell nanoparticles," J. Phys. Chem. C 119 (2015) 563.

[23] C. Burda, X. Chen, R. Narayanan, M. A. El-Sayed, "Chemistry and properties of nanocrystals of different shapes," Chem. Rev. 105 (2005) 1025.

[24] G. W. Yang, "Laser ablation in liquids: Applications in the synthesis of nanocrystals," Prog. Mater Sci. 52 (2007) 648.

[25] M. Vojkovic, D. Rayane, F. Bertorelle, R. Antoine, M. Broyer, P. Dugourd, "Synthesis of ligand-metal species by laser vaporization electrospray ionization (LAVESI)," Int. J. Mass Spectrom. 387 (2015) 45.

[26] C. P. Joshi, M. S. Bootharaju, O. S. Bakr, "Tuning properties in silver clusters," Phys. Chem. Lett. 6 (2015) 3023. 
[27] S. Kumar, M. D. Bolan, T. P. Bigioni, "Glutathione-stabilized magic-number silver cluster compounds," J. Am. Chem. Soc. 132 (2010) 13141.

[28] I. Chakraborty, A. Govindarajan, J. Erusappan, A. Ghosh, T. Pradeep, B. Yoon, R. L. Whetten, U. Landman, "The superstable $25 \mathrm{kDa}$ monolayer protected silver nanoparticle: Measurements and interpretation as an icosahedral $\mathrm{Ag}_{152}\left(\mathrm{SCH}_{2} \mathrm{CH}_{2} \mathrm{PH}\right)_{60}$ cluster," Nano Lett. 12 (2012) 5861.

[29] F. Bertorelle, R. Hamouda, D. Rayane, M. Broyer, R. Antoine, P. Dugourd, L. Gell, A. Kulesza, R. Mitrić, V. Bonačić-Koutecky, "Synthesis, characterization and optical properties of low nuclearity liganded silver clusters: $\mathrm{Ag}_{31}(\mathrm{SG})_{19}$ and $\mathrm{Ag}_{15}(\mathrm{SG})_{11}$," Nanoscale 5 (2013) 5637.

[30] B. A. Ashenfelter, A. Desireddy, S. H. Yau, T. Goodson, III, T. P. Bigioni, "Fluorescence from molecular silver nanoparticles," J. Phys. Chem. C 119 (2015) 20728.

[31] C. Noguez, A. Sanchez-Castillo, F. Hidalgo, "Role of morphology in the enhanced optical activity of ligand-protected metal nanoparticles," J. Phys. Chem. Lett. 2 (2011) 1038.

[32] B. Bousquet, M. Cherif, K. Huang, F. Rabilloud, "Absorption spectra of aryl thiol-coated silver nanoclusters: A time-dependent density-functional study," J. Phys. Chem. C 119 (2015) 4268.

[33] F. Muniz-Miranda, M. C. Menziani, A. Pedone, "DFT and TD-DFT assessment of the structural and optoelectronic properties of an organic- $\mathrm{Ag}_{14}$ nanocluster," J. Phys. Chem. A 119 (2009) 5088.

[34] J. Romann, J. Wei, M.-P. Pileni, "Computational matching of surface plasmon resonance: Interactions between silver nanoparticles and ligands," J. Phys. Chem. C 119 (2015) 11094. 
[35] M. A. Duncan, "Laser vaporization cluster sources," Rev. Sci. Instrum. 83 (2012) 041101.

[36] T. M. Ayers, J. L. Fye, Q. Li, M. A. Duncan, "Synthesis and isolation of titanium metal cluster complexes and ligand-coated nanoparticles with a laser vaporization flowtube reactor," J. Clust. Sci. 14 (2003) 97.

[37] S. Ard, C. Dibble, S. T. Akin, M. A. Duncan, "Ligand-coated vanadium oxide nanoclusters: Capturing gas phase magic numbers in solution," J. Phys. Chem. C 115 (2011) 6438 .

[38] C. A. Schneider, W. S. Rasband, K. W. Eliceiri, "NIH Image to ImageJ: 25 years of image analysis," Nature Methods 9 (2012) 671.

[39] J. Turkevich, P. C. Stevenson, J. Hillier, "A study of the nucleation and growth processes in the synthesis of colloidal gold," Faraday Discuss. 11 (1951) 55.

[40] S. Link, M. A. El-Sayed, "Spectral properties and relaxation dynamics of surface plasmon electronic oscillations in gold and silver nanodots and nanorods," J. Phys. Chem. B 103 (1999) 8426.

[41] V. Amendola, S. Polizzi, M. Meneghetti, "Free silver nanoparticles synthesized by laser ablation in organic solvents and their easy functionalization," Langmuir 23 (2007) 6766.

[42] D. Paramelle, A Sadovoy, S. Gorelik, P. Free, J. Hobley, D. G. Fernig, "A rapid method to estimate the concentration of citrate capped silver nanoparticles from UV-visible light spectra," Analyst 139 (2014) 4855.

[43] P. V. Kamat, "Photophysical, photochemical, and photocatalytic aspects of metal nanoparticles," J. Phys. Chem. B 106 (2002) 7729. 


\section{Figure Captions}

Figure 1. Electrospray ionization mass spectra of the Ag-MeCN NP sample (top) compared to that for a commercial $50 \mathrm{~nm}$ citrate capped Ag NP sample (bottom).

Figure 2. Selected TEM images of the Ag-MeCN NP sample. The left frame shows an "island" of nanoparticles left together after solvent evaporation. The right image is an expanded view of a single nanoparticle.

Figure 3. Histogram of Ag-MeCN NP sizes measured with ImageJ, summing over particles in five selected TEM images.

Figure 4. Absorption spectra of the Ag-MeCN NP sample compared to that for a commercial 50 nm citrate-capped Ag NP sample.

Figure 5. Emission spectra of the Ag-MeCN NP sample compared to that for a commercial 50 nm citrate-capped Ag NP sample, with excitation at either 220 or $400 \mathrm{~nm}$. 


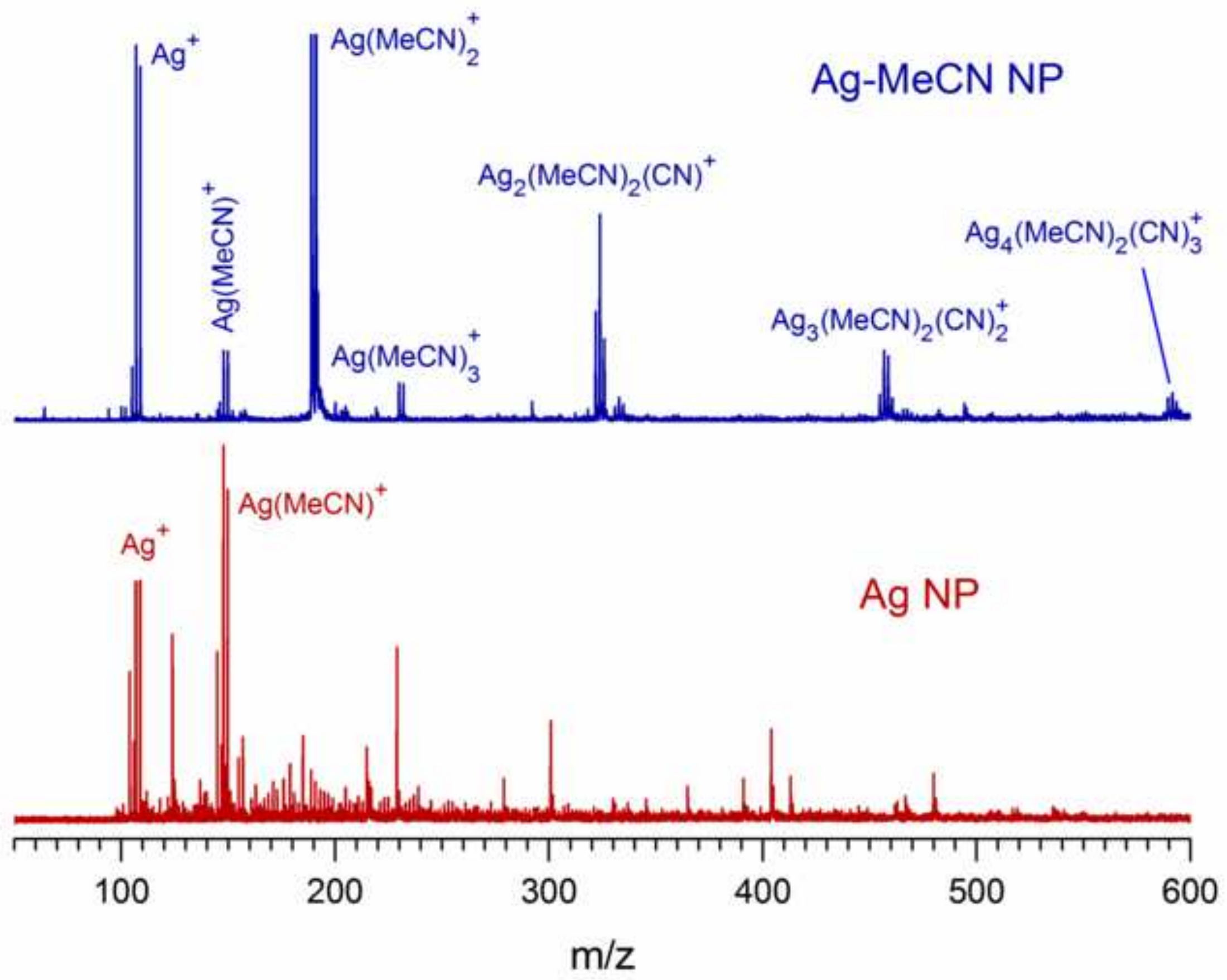



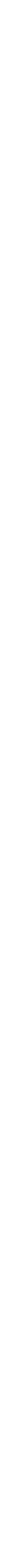

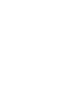
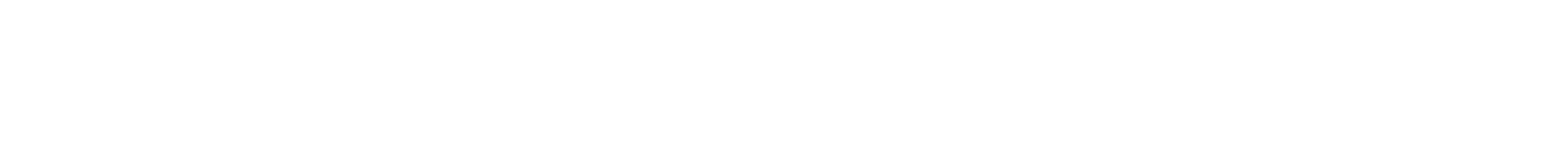


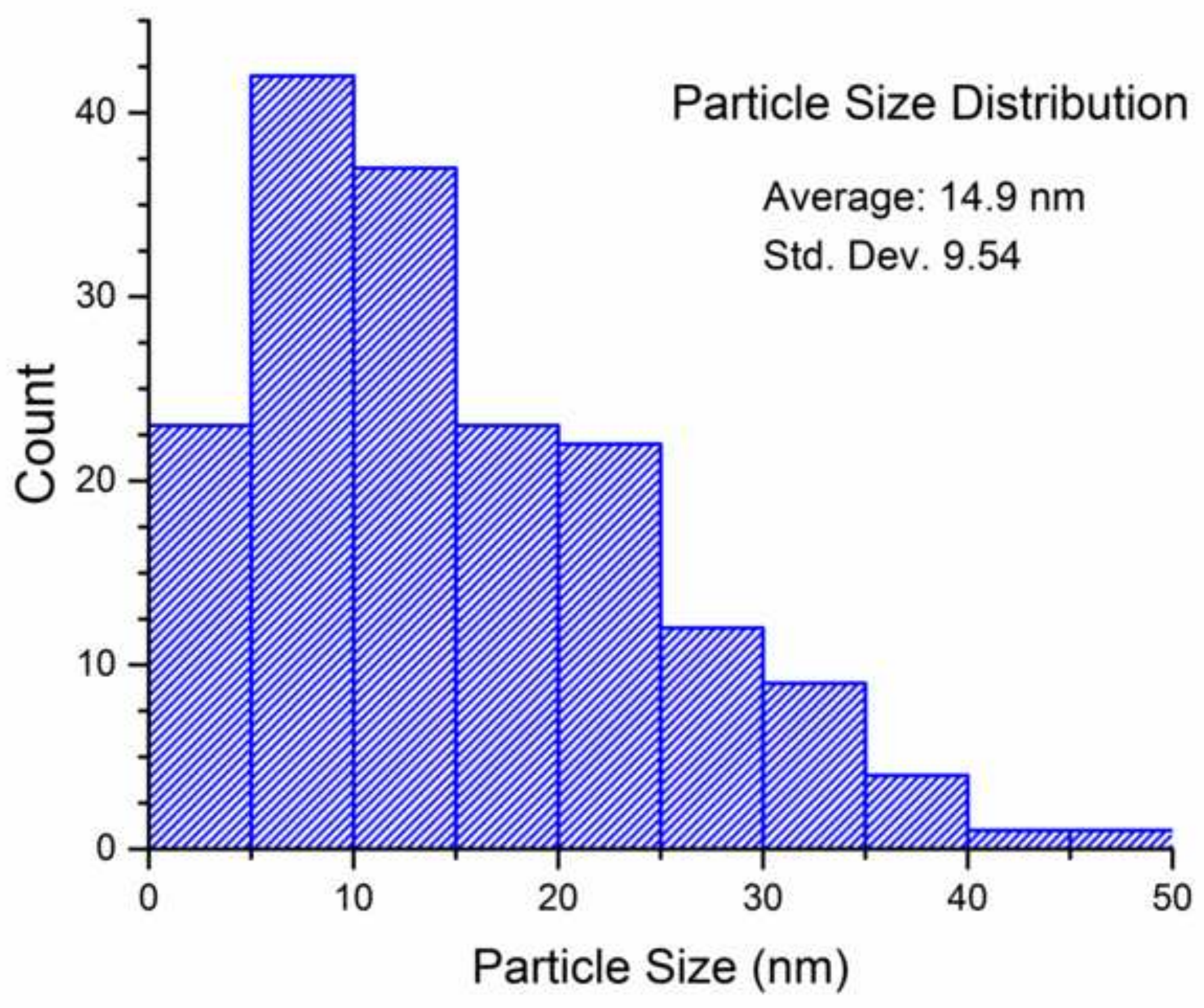




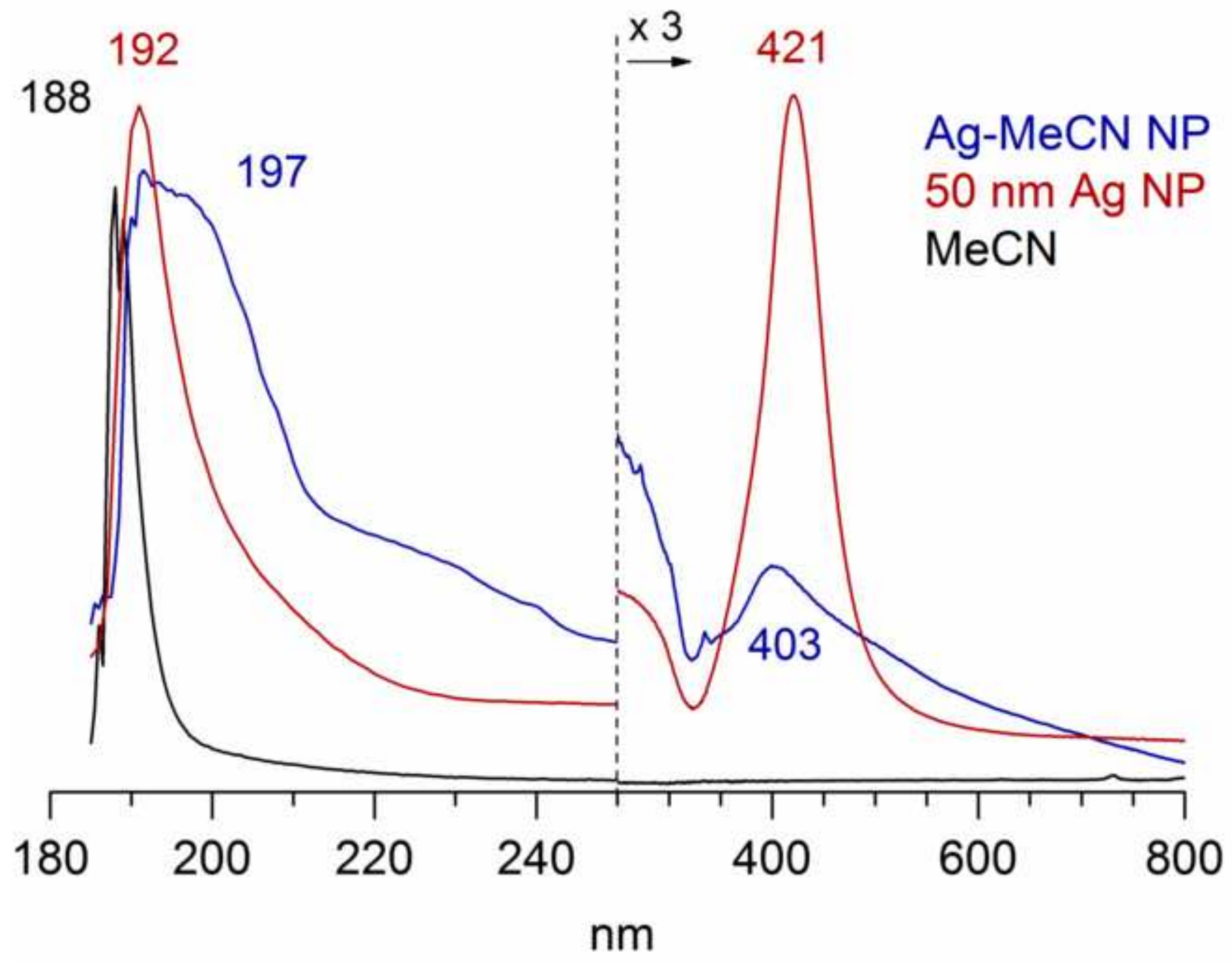




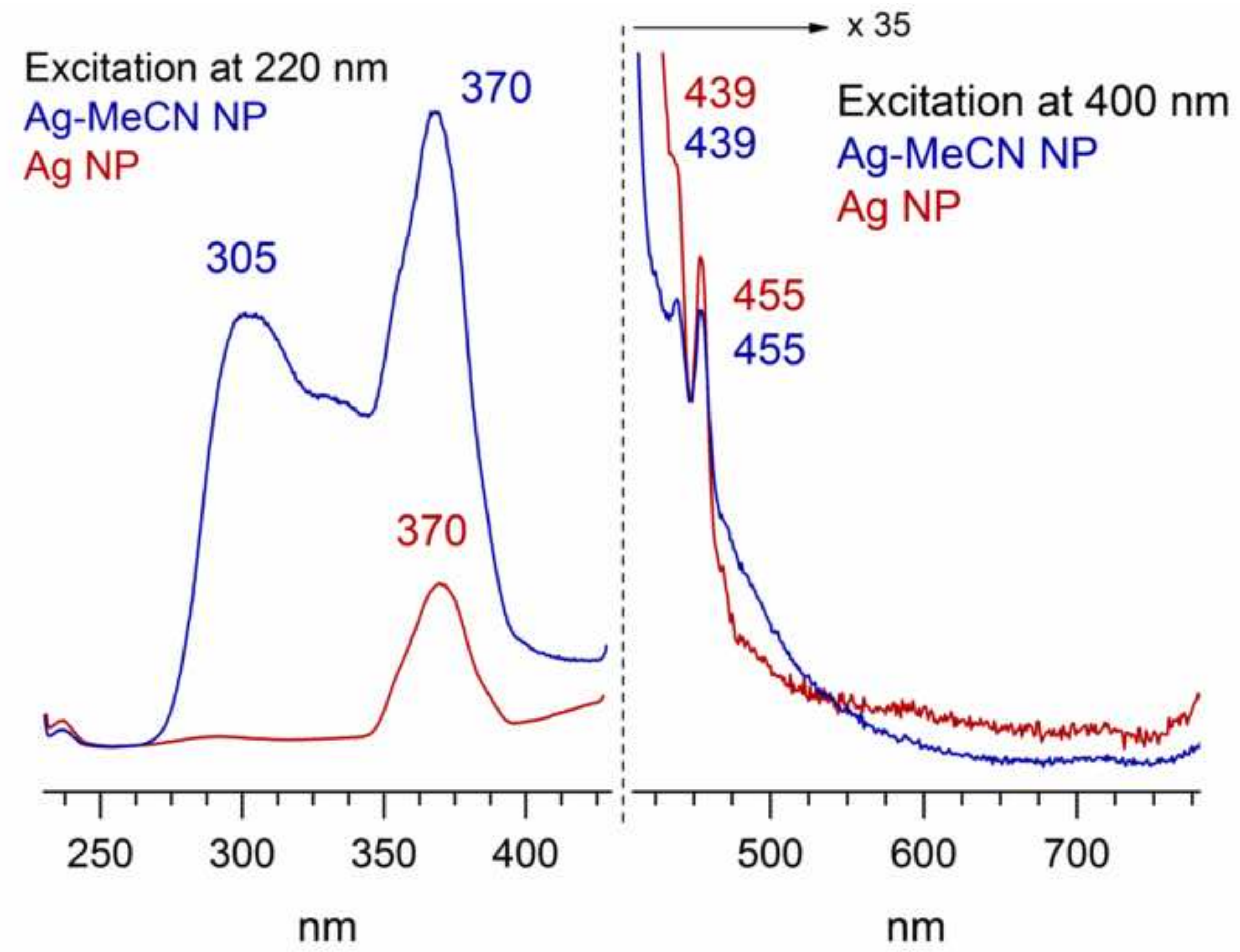

